\title{
BRAIN CONTROLLED ROBOTS FOR SEVERELY DISABLED PEOPLE AND VICTIMS OF PARALYSIS
}

\author{
Anam Atiq \\ Student, Computer Science Engineering \\ SSM College of Engineering and Technology \\ Kashmir, India
}

\author{
Seerat Hassan \\ Student, Computer Science Engineering \\ SSM College of Engineering and Technology \\ Kashmir, India
}

\author{
Mrs. Yasmeen \\ Associate Professor \\ Computer Science Engineering \\ SSM College of Engineering and Technology, Kashmir, India
}

\begin{abstract}
A Brain Controlled Interface is such an arrangement by means of which a user can be in control of an external device with regard to the brain signals. A non-invasive technique, Electroencephalography is a means to measure the activity of the brain and has proven to be compelling and efficacious prospect for the buildout of BCI. The past two decades have encountered soul stirring breakthrough in the calibre to model the brain signals that are jot down by EEG. In this paper we provide an inclusive review of how the EEG based Brain Controlled Robots can suffice as powerful abet for helping the severely disabled people and victims of paralysis to have dealings with the outside world by just their thoughts. The aerobicized users can utilise the robots by either the input devices like keyboard, mouse, joystick, touch screen or can easily operate the speech or gesture controlled robots. However, the use of assistive robots could be much mentally strenuous to use for disabled people and crippled or locked in patients of paralysis. These individuals have lost most of their muscle control making it arduous for them to exude their intensions to robots with these interfaces. But by means of these brain controlled robots natural cognitive signals can be used to control these robots means that by using this approach the severely disabled people need not to be trained in a suggested way that robots can recognise. A slick perspective like that would ameliorate the calibre of users to pilot factory robots, driverless cars, wheel chairs and many other technologies. These brain controlled robots can prove to be an efficient conveyance method for those who are not able to use verbal or gesture means such as crippled or disabled victims of paralysis and they would be able to use prosthetic devices like artificial arm, artificial leg, an automatic wheel chair just by means of their thoughts.
\end{abstract}

Keywords: Brain Computer Interface (BCI), Electroencephalography (EEG), Error Related Potentials (ErrPs), Non-Invasive BCIs, Prosthetic Devices, Assistive Robots.

\section{INTRODUCTION}

A team from MITs Computer Science and Artificial intelligence laboratory (CSAIL) and Boston University has developed a humanoid robot Baxter and by using this system, people will be enabled to correct the mistakes done by a robot in real-time using the signals generated by the brain [5]. These robots that have been developed, on the basis of brain signals generated by the human brain of the operator who is operating the robot can be used to change the working or performance of the robot. And this interesting technology can revolutionise the lives of millions of disabled people. If we take the example of crippled or paralysed people, they could be highly benefited by these types of robots as they need not to control these robots by means of gesture or speech. As the brain of paralysed people is not dead, their brain can generate signals which could afterwards alter the performance of these robots, thereby allowing them to make use of prosthetic devices, wheel chairs just by means of their thoughts. A slick approach like that could also allow the paralysed people to operate the self -driving cars seamlessly. Basically, these robots could be programmed to work a certain way for these disabled people. But the thing that is of important consideration that these brain controlled robots have been modified to work on the basis of the feedback from the brain signals of human brain. If these robots make any mistake, they can instantly rectify their mistakes because these robots will be designed in such a way that they can revamp their performance based on the thoughts of the human. This streamlined perspective can pave away for error free courtship between human and robots. Such brain storming algorithms have been deployed in this system which can perceive if the operator observes any error in the activity of the robot by identifying the brain waves in just a matter of few milliseconds. By employing such efficient brain controlled robots for the benefits of severely disabled people and victims of paralysis, they would be able to promptly tell a robot to perform a particular activity, without having an obligation of writing a command, pushing a button, perform any gestures or even saying a word.

\section{COMPARATIVE STUDY}

A Brain Controlled Interface is such an arrangement by means of which a user can be in control of an external device with regard to the brain signals [4]. A non-invasive technique, Electroencephalography is a means to measure 
the activity of the brain and has proven to be compelling and efficacious prospect for the buildout of BCI [2]. The past two decades have encountered soul stirring breakthrough in the calibre to model the brain signals that are jot down by EEG.Inspite the proof of concept was given decades ago, the well-grounded alteration of user intent into the device control commands is a major obstacle. Past work in EEG controlled robotics have necessitated training humans to think in an ordained way that computers can identify when BCIs produce movements they are much slower, less exact and less serpentine than what abled people can do easily everyday. Non-invasive BCIs that don't necessitate surgery are based on EEG recordings from the brain and have been used to manifest control of wheel chairs, robotic arms, humanoid robots etc. [1]. Much of the recent work on BCI tends to ameliorate the standard of life of severely disabled people or victims of paralysis [3]. Most robots nowadays are scheduled to follow concrete commands that permit them to carry out specific tasks. But in these brain controlled robots, the concept of EEG is used and the statistics collected from EEG monitor is used to notch up the affairs of the human brain. The system inspects for brain signals called Error Related Potential (Errps) which are triggered whenever our brain observes any fallacy [5]. As you track the robot all you have to do is cognitively agree or disagree with what it is executing. You don't have to upskill yourself to think in a definite way, the system acclimatizes to you, and not the other way around. If the robot is not convinced about its decision, it can prompt the response of the human to get a more precise, faultless and ambiguous answer. Brain control robots response to commands precipitated by brain signals rather than electronic or voice commands which could be shaky at times.

When these robots picks up the signal, it rectifies its mistake mid task. So, a feedback loop in between the human brain and the robot is established. In addition to it, another feedback loop is established if the first error was not acknowledged, that would pick up anefficacious and profound brain signal [5].It is not the first time that brain signals have been employed in controlling the robots. But the problem with that approach is that it necessitates the users to reflect in a suggested way that computers can identify. This is not an intrinsic and innate occurrence for us and can also be mentally burdensome. Natural cognitive signals can be used to control robots means that humans need not to be trained in any prescribed way, instead the system learns from just natural human thoughts.

\section{METHODOLOGY}

In these brain controlled robots the concept of EEG is used and the statistics collected from EEG monitor is used to register or notch up the affairs of the human brain. The system inspects for brain signals called Error Related Potential (Errps) which are triggered whenever our brain observes any fallacy [5]. As you track the robot all you have to do is cognitively agree or disagree with what it is executing. Success requires efficacious interaction between two adaptive controllers; the human brain which produces the brain signals that translates intent and the EEG based BCI system which renders that activity into device control commands [4].

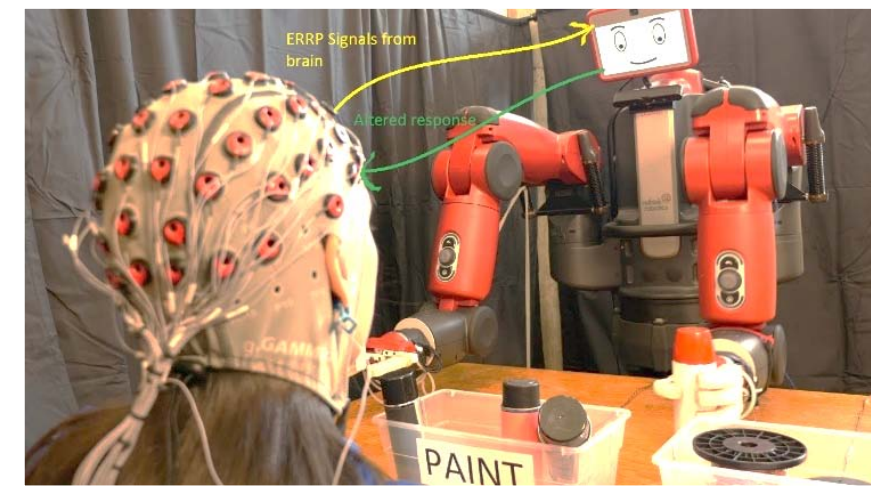

Figure 1. A feedback system between robot and human brain correcting robot action in real-time based on brain waves [5].

$\triangleright$ A user which could be a severely disabled people or victim of paralysis wears an EEG cap that quantifies their brain signals.

$\triangleright$ These brain signals are then used to ameliorate the actions of robot.

$\triangleright$ If the robot discerns these signals, the robot changes its actions.

$\gg$ If not, the robot pursues with its planned activity.

$\triangleright$ This system works in real-time identifying the signals generated by brain within millisecond.

\section{ADVANTAGES}

$\triangleright \quad$ It has repercussions for future for performing more complex tasks.

$\triangleright$ This system could also have big repercussions for people who can't communicate by verbal means.

$\triangleright$ It could revolutionize the lives of immobilised or ever locked in victims of paralysis.

$\triangleright$ It could also be used to operate autonomous cars.

$\triangleright$ The robot-human interaction provide a means of blunt and steadfast communication.

$>$ It is not mentally stringent as it does not require humans to be trained in a specific manner.

$\triangleright$ It could be used to control the prosthetic devices just by means of thoughts.

$\triangleright$ It can be used by paralysed patients to use a tablet wirelessly.

$\triangleright$ Brain control robots response to commands precipitated by brain signals rather than electronic or voice commands which could be shaky at times.

\section{DISADVANTAGES}

$\triangleright$ It is very difficult to fabricate these systems swift and steadfast enough to be of practical use in the real world.

$>\quad$ There could be a complication for damage as brain tissue is pliable and soft.

$>$ These demos have been used in labs where the rooms are hushed, the technical setup is extended, coherent and methodical.

$\triangleright$ It produces movements that are much prolonged, less explicit and less tortuous than what able bodied people do easily everyday. 


\section{CONCLUSION}

We conclude this paper with the contemporaneous challenges and upcoming research inclination. EEG based Brain Controlled Robots can suffice as powerful abet for helping the severely disabled people and victims of paralysis to have dealings with the outside world by just their thoughts. Researchers have already been able to use the brain signals to control a robotic arm and have also been successful in extricating the movement intent of paralysed patients with the help of their brain waves to operate a tablet wirelessly. But the well-grounded alteration of user intent into the device control commands is a major obstacle and can prove to be very challenging. Give how strenuous it can be for the disabled people to communicate their intensions to the robots by verbal or gesture means, work in this area would have profound footprint on the upcoming doom of collaboration between assistive robots and paralysed people. Completely employing this technology to improve the quality of life of paralysed people can totally transmute the lives of millions of disabled people because this type of technology does not require the operators to think in a endorsed way as these robots operate on the basis of brain signals generated by the human brain. Thus enabling the paralysed people to correct the mistakes done by the robot in real-time because these robots are designed in such a way that they can revamp their performance based on the thoughts of the human without causing much mental stress. But it is very difficult to fabricate these systems swift and steadfast enough to be of practical use in the real world and there could be a complication for damage as brain tissue is pliable and soft.

\section{REFERENCES}

[1] L. Bi, X. Fan, and Y. Liu, "EEG-based brain-controlled mobile robots: A survey,” IEEE Trans. Human Mach. Syst., vol. 43, no. 2, pp. 161-176, Mar. 2013.
[2] S. Moghimi, A. Kushki, A. Marie, and T. Chau, “A review of EEG-based brain-computer interfaces as access pathways for individuals with severe disabilities,” Assist. Technol., vol. 25, pp. 99-110, 2013.

[3] H.-J. Hwang et al., "EEG-based brain-computer interfaces (BCIs): a thorough literature survey,” Int. J. Hum. Comput. Int. 29(12), 814- 826 (2013)

[4] B. Blankertz, K.-R. Müller, D. Krusienski, G. Schalk, J.R. Wolpaw, A. Schlögl, G. Pfurtscheller, J. del R. Millán, M. Schröder, and N. Birbaumer, "The BCI competition III: Validating alternative approachs to actual BCI problems," IEEE Trans. Neural Syst. Rehab. Eng., vol. 14, no. 2, pp. 153159, 2006.

[5] A. F. Salazar-Gomez, J. DelPreto, S. Gil, F. H. Guenther, and D. Rus, "Correcting robot mistakes in real time using eeg signals,” in ICRA. IEEE, 2017.

\section{BIOGRAPHIES}

AnamAtiq, is pursuing B.E Degree from SSM College of Engineering \& Technology in Department of Computer Science Engineering, University of Kashmir, J\&K, India. Fields of interest are JAVA, Artificial Intelligence and Robotics.

Seerat Hassan, is pursuing B.E Degree from SSM College of Engineering \& Technology in Department of Computer Science Engineering, University of Kashmir, J\&K, India. . Fields of interest are JAVA, Artificial Intelligence and Robotics.

Mrs. Yasmeen, is the Associate Professor at SSM College of Engineering and Technology in Department of Computer Science Engineering, University of Kashmir, J\&K, India. Fields of interest are Computer Graphics, Data Mining and Networking. 